


\title{
Research Paper \\ Identifying Some Risk Factors for the Time to Death of the Elderly Using the Semi- Parametric Blended Model of Survival Analysis With Competing Risks
}

\author{
Samane Hajiabbasi ${ }^{1}$, ${ }^{*}$ Mehdi Rahgozar ${ }^{1}$, Akbar Biglarian ${ }^{1}$, Arash Jalali ${ }^{2}$, Mohammad Javad Azadchehr ${ }^{1}$
}

1. Department of Biostatistics, University of Social Welfare and Rehabilitation Sciences, Tehran, Iran.

2. Tehran Heart Center, Tehran University of Medical Sciences, Tehran, Iran.

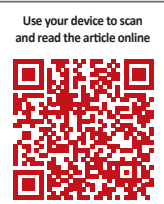

dration: Hajiabbasi S, Rahgozar M, Biglarian A, Jalali A, Azadchehr MJ. [Identifying Some Risk Factors for the Time to Death of the Elderly Using the Semi-Parametric Blended Model of Survival Analysis With Competing Risks (Persian)]. Iranian Journal of Ageing. 2018; 12(4):518-527. https://doi.org/10.21859/SIJA.12.4.518

Received: 24 Aug. 2017 Accepted: 18 Nov. 2017

Key words:

Aging, Semiparametric blended model, Competing risks, Survival analysis

\section{A B STRACT}

Objectives As the population of elderly people in Iran is rising, determining the risk factors of their death is necessary. The purpose of this study was to identify the risk factors that reduce the survival time of elderly people.

Methods \& Materials In a longitudinal retrospective study, data of 510 elderly people aged over 60 years, who were admitted to Kashan's Golabchi nursing home from 2000 to 2012 were collected and analyzed. To identify some risk factors of time to death in elderly, semi-parametric mixture competing risk model in survival analysis was fitted to the data. To estimate the model parameters, Expand-Maximize-Compress (EMC) algorithm was used and parameters and their $95 \%$ confidence intervals were estimated using $\mathrm{R}$ software (version 3.3.1).

Results In separate one-variable fitted models, the variables like high blood lipids ( $\widehat{\mathrm{HR}}=1.04 ; \mathrm{Cl}=1.00$, 1.31), history of myocardial infarction $(\widehat{H R}=0.90 ; C l=1.04,1.10)$, stroke history $(\widehat{H R}=0.95 ; C l=1.00,1.14)$, and deaths of elderly people with cardiovascular diseases were significant. In the fitted multivariate model, renal problems had a significant effect ( $\widehat{H R}=1.58 ; \mathrm{Cl}=1.77,2.83)$ on time to death of elderly.

Conclusion In single-variable fitting, age, history of myocardial infarction, history of stroke, and kidney problems were identified to have significant effects on the time to death of the elderly. Based on onevariable semi-parametric competing risk mixture fitted models, more significant risk factors for the time to death of elderly was identified when compared with a fitted multivariate mode to the data. This implies that the role of some independent variables can be explained by other independent variables.

\section{Extended Abstract}

\section{Objectives}

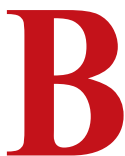

ecause the elderly population is increasing in Iran, awareness of various causes of death in the elderly is necessary. Certainly, with an increase in the number of elderly people, the mortality rate in the community will be on the rise, followed by the increase in mortality rates in this age group [1].

The present study aims to identify the risk factors that reduce the survival time of the elderly so that preventive measures could be considered in the identification, clinical trials, and therapeutic measures to eliminate serious risks and increase survival time for the elderly. The main purpose of this study was fitting a semi-para-

\section{* Corresponding Author:}

Mehdi Rahgozar, PhD

Address: Department of Biostatistics, University of Social Welfare and Rehabilitation Sciences, Tehran, Iran.

Tel: +98 (21) 22180146

E-mail: m_rahgozar2003@yahoo.com.au 
metric blended model of survival analysis with competing risks for the elderly living in the nursing home and then estimating the parameters. Deaths in the elderly people due to cardiovascular diseases and other causes have been considered as competing risks.

\section{Methods \& Materials}

The research method was retrospective. Analysis of data was performed by studying 510 elderly people over 60 years of age, who were admitted to Kashan's Golabchi nursing home from 2000 to late 2012. Independent variables related to the time to death of the elderly included gender, age at the start of admission, blood pressure, blood lipids, mobility status, history of myocardial infarction, history of stroke, and kidney problems. The dependent variable was the length of stay at the nursing home, which was calculated from the difference between the admission date and the discharge date [2]. Heterogeneity of patients is often ignored in the analysis of medical data. On the other hand, individual treatment is often important in medical sciences; however, the use of blended statistical models to analyze data related to a sample of the heterogeneous population can lead us to a proper analysis [3]. Another feature of this method is the lack of the need for the presumption of the independence of competing risks and the simultaneous estimation of parameters [4-6]. A semi-parametric blended model of survival analysis with competing risks was used to analyze the data, and for this the expectation-conditional maximization (ECM) algorithm was applied to estimate the model parameters [7].

In the model, the decision criterion for the significance of indicators of odds ratio and risk ratio is confidence intervals. The collected data were first analyzed separately as single-variable for each independent variable and again as multivariate by entering all independent variables in the model. The target incident was the death of the elderly, and those elderly who had not died by the end of the study were considered as censored data based on the time variable. Deaths due to cardiac and non-cardiac causes were defined as competing risks. The present research was approved by the ethics committee of the University of Social Well-being and Rehabilitation Sciences (IR.USWR.REC.1394.241). Data analysis was performed using R 3-3-3 software.

\section{Results}

Of 510 elderly people living in Golabchi nursing home of Kashan, 29\% (148 individuals) were men, and
71\% (362 individuals) were women. Also, 19.4\% (99 cases) of them had died of cardiac causes and $44.1 \%$ (225 cases) due to non-cardiac causes. The remaining $36.5 \%$ of the elderly (186 people) were either survived or discharged by the end of the study, which was considered as censored data in the study. The median age of the elderly was 11.18 years, which was used as the grouping level in the age variable. The mean age of the elderly was 80.35 years with a standard deviation of 18.19 years.

In the single-variable fitting of the models, factors such as blood lipids $\{(\mathrm{CI}=1.00 / 1,1.31) ; \widehat{\mathrm{HR}}=1.04\}$, history of myocardial infarction $\{(\mathrm{CI}=1.04,1.10)=\mathrm{CI}$; $90.0=\widehat{\mathrm{HR}}\}$, history of stroke $\{(\mathrm{CI}=1.00,1.14)$; $\widehat{\mathrm{HR}}$ $=0.95\}$ had a significant effect on the time to death of elderly with cardiovascular diseases. The coefficients derived from the univariate models in the estimation of the target incidence ratio, the chance of dying of cardiac diseases in the elderly men were $60 \%$ more than the elderly women $(\mathrm{OR}=0.66)$, with a confidence interval for this odds ratio $\mathrm{CI}=0.55,1.03$. The risk of death due to cardiac diseases in elderly patients with the history of myocardial infarction was 2.61 times more than others $(2.61=\mathrm{OR}$ experience of myocardial infarction). In a single-variable model, for an elderly person with a history of heart attack, the probability of death due to cardiac causes is 0.52 , and the probability of non-cardiac death in an elderly person with a history of heart attack is 48.0 .

In the fitting of the multivariate model (fitting a model with 8 independent variables simultaneously), renal problems have a significant effect $(\mathrm{CI}=1.77,2.83$; $\widehat{\mathrm{HR}}$ $=1.58$ ). The probability of cardiac and non-cardiac deaths was 0.17 and 0.83 , respectively, in the elderly men under 81.11 years old who have high blood fat along with abnormal motor status, high blood pressure, and no history of myocardial infarction and renal failure. Also, the probability of death due to cardiac causes in elderly women with a minimum age of 81.11 years who have high blood fat along with abnormal motor status and high blood pressure and have no history of myocardial infarction and renal failure. It is while the probability of non-cardiac deaths in these women is 0.41 .

In the fitting of a multivariate model with the constant effect of other variables, the probability of cardiac deaths is lower in men than in women $(\mathrm{OR}=0.79)$, with a confidence interval of $0.84,0.97$. In addition, with the constant effect of other variables, the risk of cardiac deaths in elderly with renal problems is 1.58 times great- 
er than the elderly without renal problem $(\widehat{\mathrm{HR}}=1.88)$ with a confidence interval of $1.77,2.83$.

\section{Conclusion}

In single-variable fittings, the effects of the factors including age, history of myocardial infarction, history of stroke, and renal problems on time to death of the elderly were identified. Also, the results of multivariate analysis showed that with the constant effect of other variables, the renal problems variable has a significant effect on the time to death of the elderly living in nursing home. Therefore, it is recommended to consider preventive processes in the identification, clinical trials, and therapeutic measures to eliminate serious risks and to increase survival time for the elderly.

In the current aging society, proper planning for preventing renal and motor problems as well as good nutrition can help the quality of life of the elderly. One of the limitations of this study was the lack of accurate patient information, which might have resulted in the insignificant effects of some important clinical variables.

\section{Acknowledgments}

This research was extracted from the MSc. thesis of the first author in the Department of Biostatistics, University of Social Welfare and Rehabilitation Sciences, Tehran.

\section{Conflict of Interest}

The authors declared no conflicts of interest. 


\title{
شناسايى برخى عوامل خطر زمان تا مركَ سالمندان، با بهكارتيرى مدل آميخته نيمهإرامترى مخاطرات رقيب تحليل بقاس برح
}

\author{
سمانه حاجى عباسى'، "مهدى رهكذر'، اكبر بيكلريان'، آرش جلالى'، محمدجواد آزادجهر' \\ ا - إكروه آمار زيستى، دانشكاه علوم بهزيستى و توانبخشى، تهران، ايران.

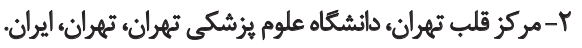

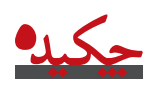

الهداف با توجه به افزايش جمعيت سالمندان ايران، آشنايى با علل مختلف در مرك سالمندان ضرورى الست. هدف اين مطالعه شناسايى

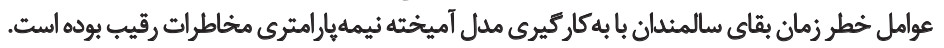

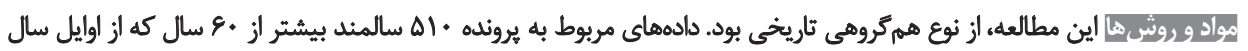

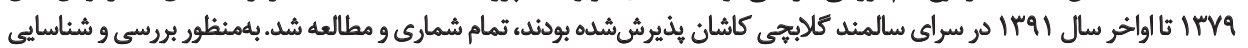

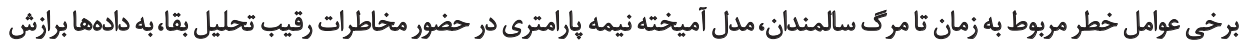

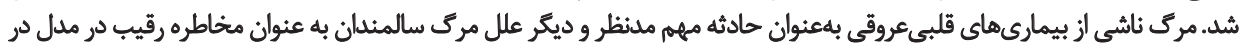

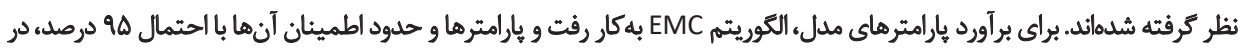

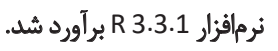

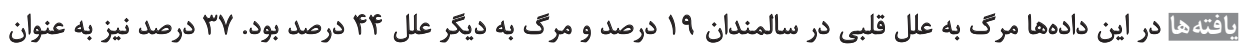

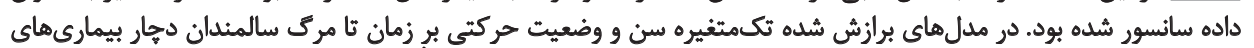

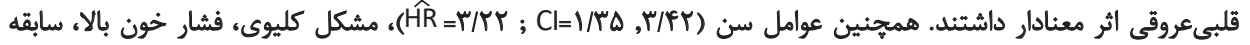

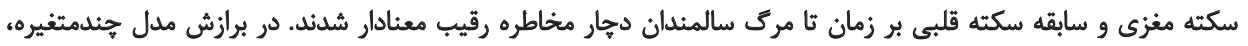

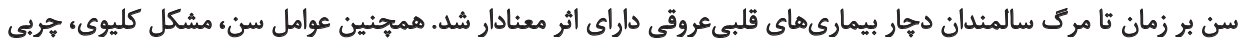

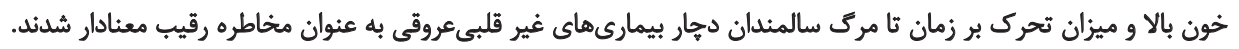

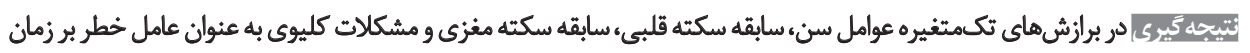

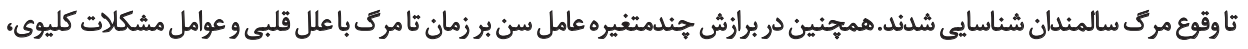

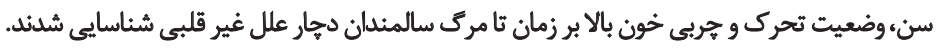

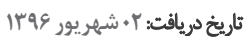

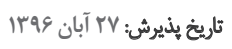

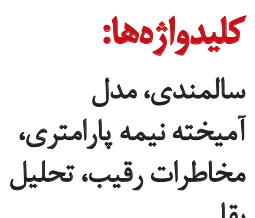

بقاما

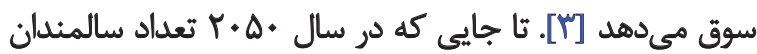

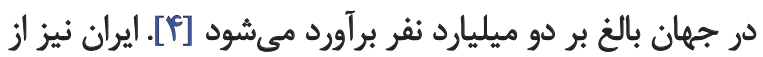

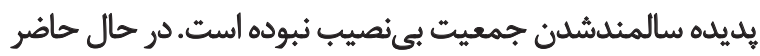

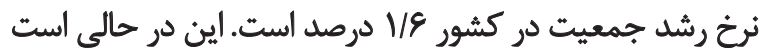

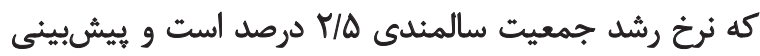

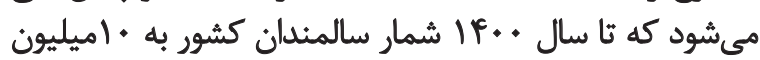

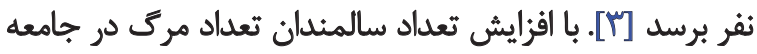

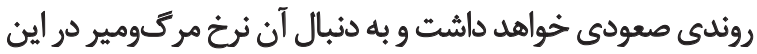
كروه سنى صعودى خواهد شوند.

بيشترين علت مرك در بين سالمندان ناشى از بيمارىهاى

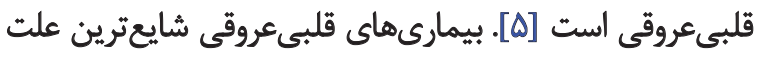

doleo

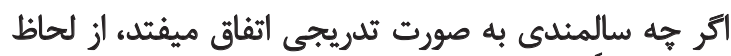

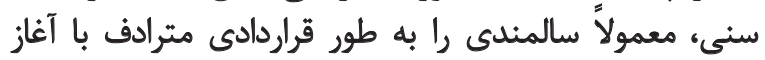

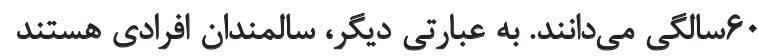

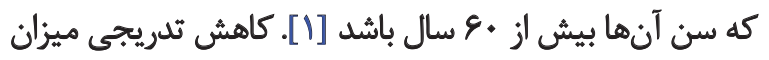

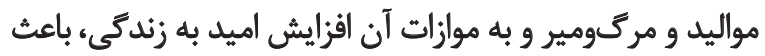

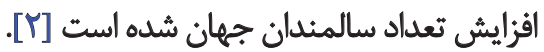

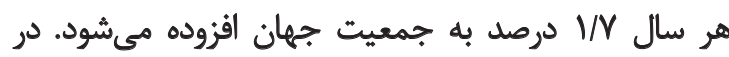

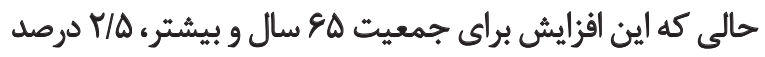
است. اين تركيب سنى، جمعيت جهان رابث رابه سوى سالمند شدن

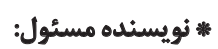

$$
\begin{aligned}
& \text { دكتر مهدى رهكذر } \\
& \text { نشائى: تهران، دانشكاه علوم بهزيستى و توانبخشى، كروه آمار زيستى. }
\end{aligned}
$$

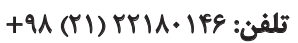

$$
\begin{aligned}
& \text { يست الكترونيكى: m_rahgozar2003@yahoo.com.au }
\end{aligned}
$$


زندهماندن ثا إيايان مطالعه را تجربه كرده بودند. مرك به بهعنوان

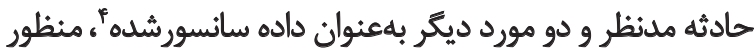

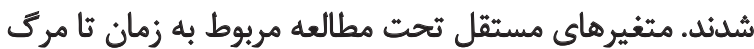

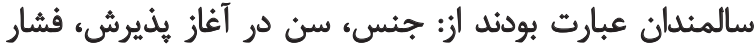

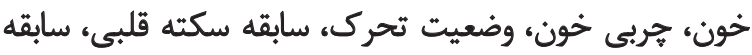

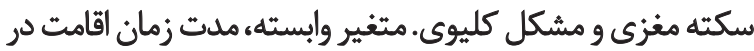

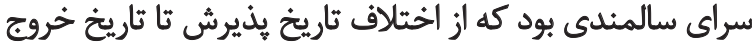

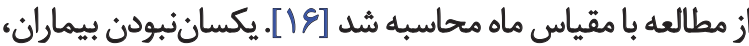

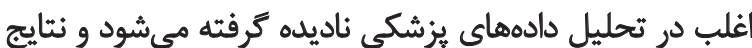

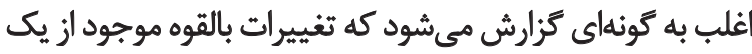
بيمارى به بيمار ديكر اغلب ناديده گرفته مي مُشود.

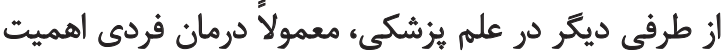

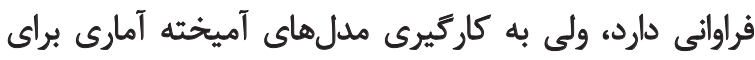

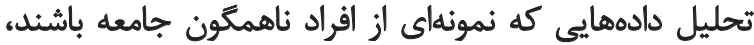

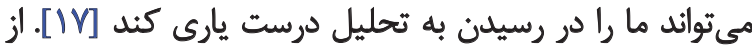

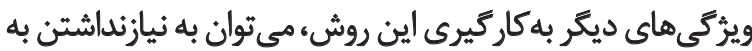

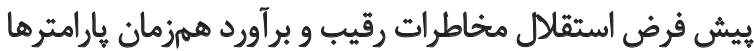

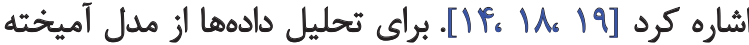

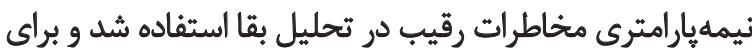

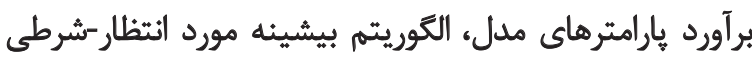

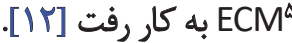

در مدل بهكارففته، معيار تصميمگيرى براى معنادارشدن

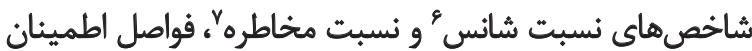

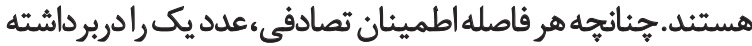

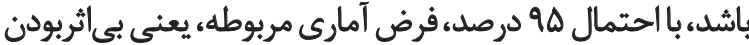

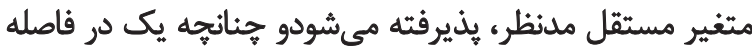

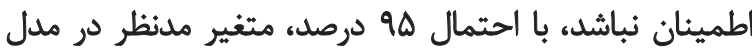

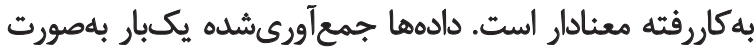

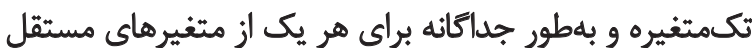

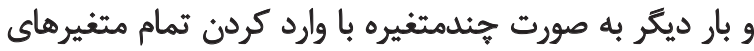

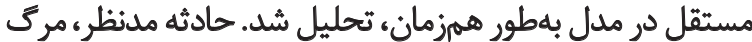

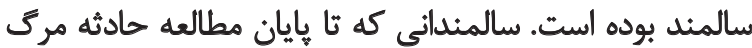

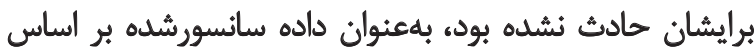

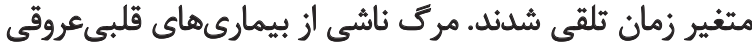

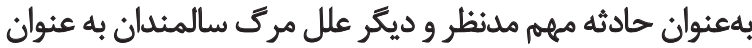

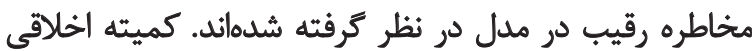

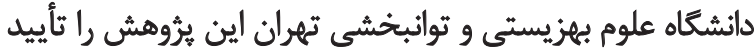

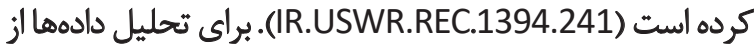

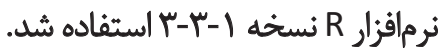

4. Censored Data

5. Expectation Conditional Maximization

6. Odds ratio

7. Hazard ratio
مركىمير در اكثر كشورهاى جهان است [؟]. اين بيمارىها

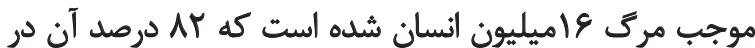

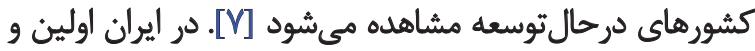

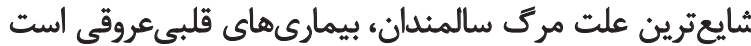

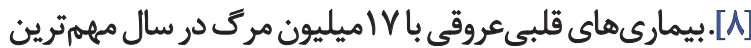

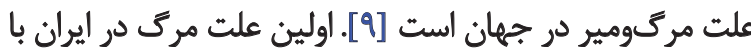

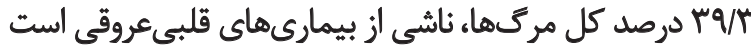

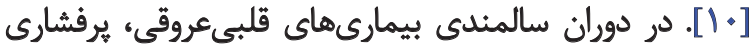

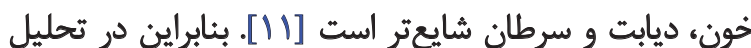

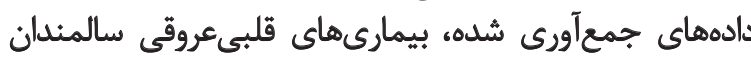

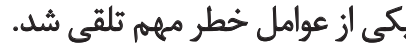

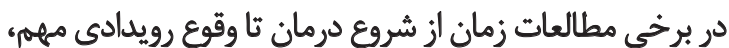

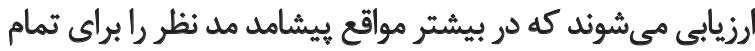

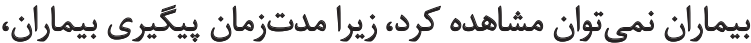

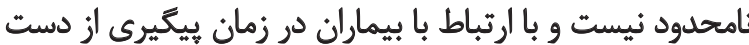

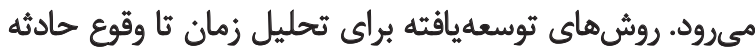

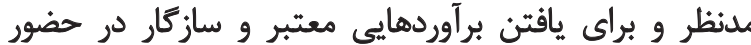

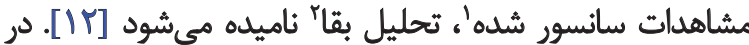

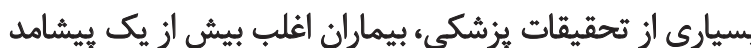

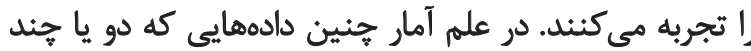

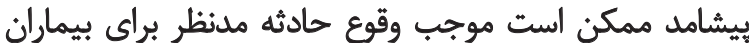

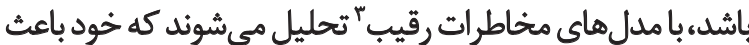

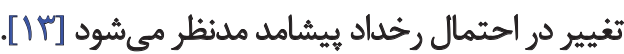
از طرفى مدل هاى آميخته بقا از جمله روشهاي آمارى هستند

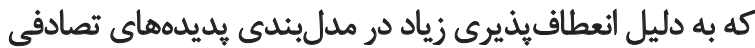

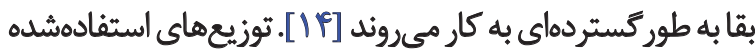

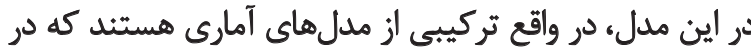

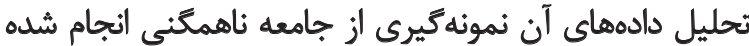

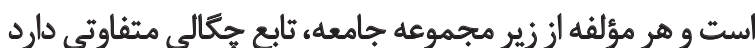

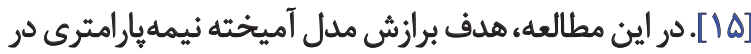

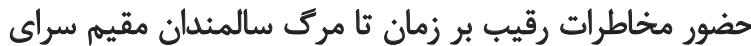
سالمندى و برآورد يارامترها بودي بر است.

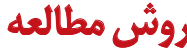

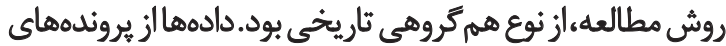

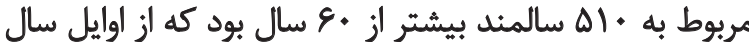

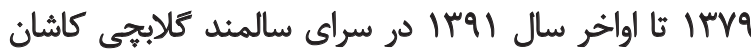

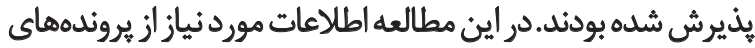

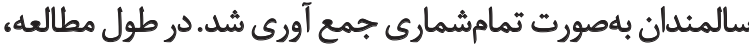

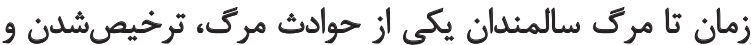

1. Censored observations

2. Survival analysis

3. Competing risks 


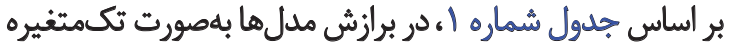

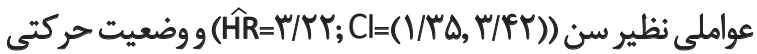

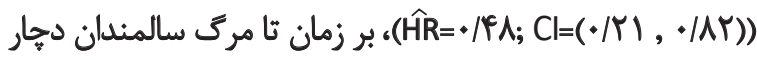
بيمارى هاى قلبي-عروقى داراى اثر معنادار بودند.

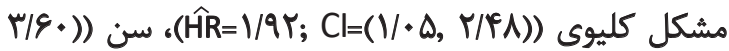

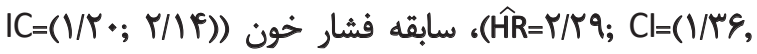

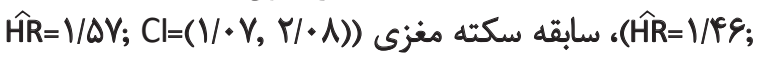

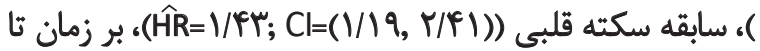
مركى سالمندان با علل غير قلبى داراى اثر معنادار بودند.

همجنين در بررسى مدل بلصورت تك متغيره، براى فرد

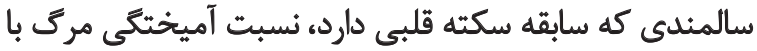

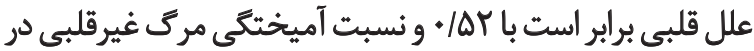

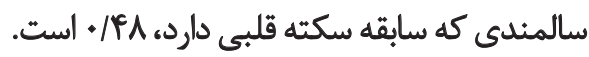

يافتهها

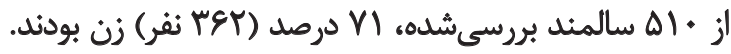

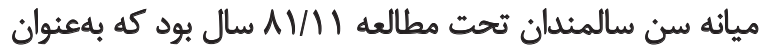

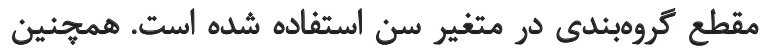

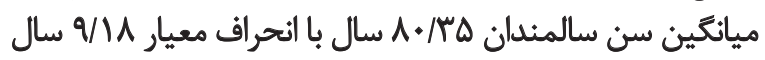

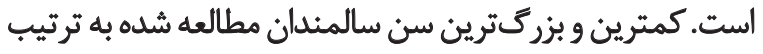

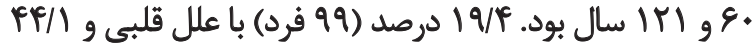

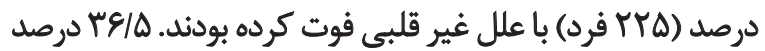

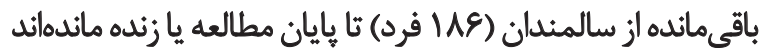

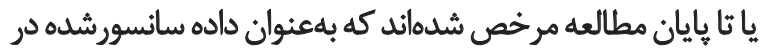

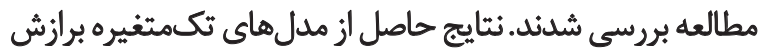
شده در جدول شماره ال درج شده است.

جدول ا. برآورد إبارمترها در مدل نيمهيار امترى آميخته ريسكهاى رقابتى برازش شده به دادهاى سالمندان كاشان در تحليلهاى تكمتغيره

\begin{tabular}{|c|c|c|c|c|c|c|c|c|}
\hline \multicolumn{4}{|c|}{ برآورد هار امترهاى ثابع بقا } & \multicolumn{4}{|c|}{ برآورد بار امثرهاى نسبث أميختيّكى در مدل آميخخته } & \multirow[b]{2}{*}{ متغيرها } \\
\hline 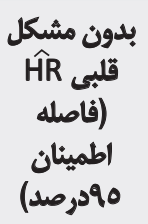 & بدون مشكل & 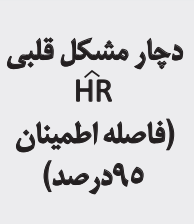 & 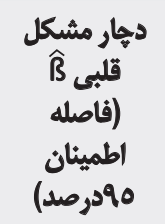 & 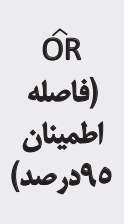 & موردمينان) & 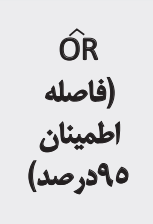 & 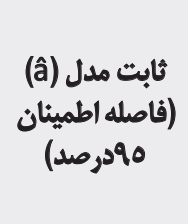 & \\
\hline $\begin{array}{c}\cdot / V 1 \\
(\cdot / 8), 1 / 4 \lambda)\end{array}$ & $\begin{array}{l}-. / r \Delta \\
. / 1 \% q) \\
(-. / 4 q\end{array}$ & $\begin{array}{c}V / 8 . \\
(\cdot / \Delta V, Y / Q Y)\end{array}$ & $\begin{array}{c}\cdot / p V \\
(-\cdot / \Delta V, V / \cdot V)\end{array}$ & $\begin{array}{l}\cdot / 8 \\
\mid / \& A) \\
(\cdot / r A\end{array}$ & $\begin{array}{c}-. / \Delta 1 \\
(-. / 98, \cdot / \Delta Y)\end{array}$ & $\begin{array}{c}. / P r \\
(\cdot / F), \cdot / \Delta A)\end{array}$ & $\begin{array}{c}-. / N Q \\
(-+/ 9 \cdot,-\cdot / \Delta \varphi)\end{array}$ & جنسيت \\
\hline $\begin{array}{c}\text { r/rq } \\
(1 / r q, r / q \cdot)\end{array}$ & $\begin{array}{c}\cdot / A N^{\prime} \\
(\cdot / M I, I / M A)\end{array}$ & $\begin{array}{c}r / T r \\
(N / T \Delta, r / R T)\end{array}$ & $\begin{array}{c}\text { V/Y } \\
(\cdot / r \cdot, V / M)\end{array}$ & $\begin{array}{l}\cdot / \Delta \Delta \\
1 / T E) \\
(+/ R T\end{array}$ & $\begin{array}{c}-. / \Delta 9 \\
(-\cdot / A F, \cdot / M T)\end{array}$ & $\begin{array}{c}1 / I V \\
(\cdot / T q, V / M)\end{array}$ & $\begin{array}{c}\cdot / \mathbb{} \\
(-1 / \pi, \cdot / R T)\end{array}$ & سن \\
\hline $\begin{array}{c}\text { VRr" } \\
(\cdot / A 9, r / T V)\end{array}$ & $\begin{array}{l}. / N E \\
. / A Y) \\
(-. / N Y\end{array}$ & $\begin{array}{c}V / * f \\
(* / N \&, V / r+)\end{array}$ & $\begin{array}{c}* / * f \\
(-* / T V, * / M)\end{array}$ & $\begin{array}{l}1 / \cdot 8 \\
1 / m 4) \\
(0 / 99\end{array}$ & $\begin{array}{c}+1+8 \\
(-+1+1,+/ 79)\end{array}$ & $\begin{array}{c}\cdot / \pi T \\
(\cdot / \pi \&,+/ \Delta \Delta)\end{array}$ & $\begin{array}{c}-\cdot / A r \\
(-1 /+r, \cdot / \Delta q)\end{array}$ & خُرن \\
\hline $\begin{array}{c}\text { V/ar } \\
(1 / \cdot \Delta, r / F A)\end{array}$ & $\begin{array}{c}. / 80 \\
(+1+\Delta, \cdot / 91)\end{array}$ & $\begin{array}{c}1 / P A \\
(\cdot / Q Y, Y / M Y)\end{array}$ & $\begin{array}{c}. / \% q \\
(-* / \cdot r, \cdot /(\Delta))\end{array}$ & $\begin{array}{l}. / V \\
V / M Y) \\
(\cdot / 4 A\end{array}$ & $\begin{array}{c}-. / r \Delta \\
(-\cdot / M+, / N T)\end{array}$ & $\begin{array}{c}. / P a \\
(\cdot|+|,+|F|)\end{array}$ & $\begin{array}{c}-\cdot / v 9 \\
(-\cdot / 9 \cdot,-\cdot / \Delta \cdot)\end{array}$ & كليوى \\
\hline $\begin{array}{c}.181 \\
(\cdot / \Delta, r, r / r)\end{array}$ & $\begin{array}{l}-. / 4 \\
. / N 1) \\
(-. / 89\end{array}$ & $\begin{array}{c}\cdot / / A \\
(\cdot / Y), \cdot(A Y)\end{array}$ & $\begin{array}{l}-\cdot / M \\
-\cdot / r \cdot) \\
(-1 / \Delta A\end{array}$ & $\begin{array}{l}\text { I/FQ } \\
T / T \Delta) \\
(\cdot / 91\end{array}$ & $\begin{array}{c}\cdot / m V \\
(-* / \cdot 9, \cdot / A 1)\end{array}$ & $\begin{array}{c}\cdot / r r \\
(\cdot / r \cdot, \cdot / P A)\end{array}$ & $\begin{array}{c}-. / 99 \\
\left(-1 / 19,-\cdot / 7^{6}\right)\end{array}$ & رضعيت \\
\hline $\begin{array}{c}1 / f 8 \\
(1 / T \cdot, r / N F)\end{array}$ & $\begin{array}{c}\cdot /{ }^{\prime} A \\
(\cdot / M, \cdot / N 8)\end{array}$ & $\begin{array}{c}\cdot / M 1 \\
(\cdot / N e, 1 / M r)\end{array}$ & $\begin{array}{c}-\cdot / \pi f \\
(-1 / \cdot r, \cdot / T)\end{array}$ & $\begin{array}{l}r / M T \\
r / T \Delta) \\
(1 \cdot / \Delta V\end{array}$ & $\begin{array}{c}* / \Lambda \\
(* / F \Delta, \mid / M)\end{array}$ & $\begin{array}{c}\cdot / r q \\
(\cdot / r \Delta, \cdot / m q)\end{array}$ & $\begin{array}{c}-1 / \pi f \\
(-1 / F \cdot,-1 / \cdot 1)\end{array}$ & سابقه فشار \\
\hline $\begin{array}{c}V / \Delta V \\
(V / \cdot V, r / \cdot 1)\end{array}$ & $\begin{array}{c}\cdot / \% \Delta \\
\left(* / * V, * / M^{\Psi}\right)\end{array}$ & $\begin{array}{c}. / 90 \\
(* / N 9,1 / 19)\end{array}$ & $\begin{array}{c}-\cdot / \cdot \Delta \\
(-+/ T F,+/ \lambda \Delta)\end{array}$ & $\begin{array}{l}r / 1 r \\
r / N e) \\
(\circ / 9 q\end{array}$ & $\begin{array}{c}1 / 16 \\
(-+1+1,1 / \% \Delta)\end{array}$ & $\begin{array}{c}\cdot / M T \\
(\cdot / K A,+/ \Delta S)\end{array}$ & $\begin{array}{c}-1 / 10 \\
(-1 \cdot / M V,-+/ \Delta \Lambda)\end{array}$ & سابقه \\
\hline $\begin{array}{c}\text { V/FT } \\
(1 / / 9, r / F I)\end{array}$ & $\begin{array}{c}. / F e \\
(* / / V, \cdot / M)\end{array}$ & $\begin{array}{c}\cdot / \pi \\
(\cdot / / \%,, / / \Delta)\end{array}$ & $\begin{array}{c}-1 / 1 . \\
(-1 / \pi+,+/ / 4)\end{array}$ & $\begin{array}{l}r / q) \\
r / m q) \\
(+N+,\end{array}$ & $\begin{array}{c}. / Q \Phi \\
(-* / \% \Delta, V / T Y)\end{array}$ & $\begin{array}{c}\cdot / \pi \Delta \\
(\cdot / \pi r, \cdot / \pi q)\end{array}$ & $\begin{array}{c}-1 / \cdot f \\
(-1 / 11,-\cdot / M)\end{array}$ & سكته قلبى سابقه \\
\hline
\end{tabular}


جدول T. برآورد هارامترها در مدل نيمهايارامترى آميخته ريسكهاى رقابتى در تحليل جيند متغيره

\begin{tabular}{|c|c|c|c|c|c|c|c|c|c|c|c|c|}
\hline \multicolumn{8}{|c|}{ برآورد بارامثرهاى تابع بقا } & \multirow{2}{*}{\multicolumn{4}{|c|}{ برأورد هارامترهاي نسبت أميختكى در مدل }} & \\
\hline & ل & بدون مشيك & & & كل قلبيى & يجاهار مئ & & & & & & \\
\hline فاصله & $\hat{H R}$ & فاصله & $\widehat{\beta}$ & فاصله & $\hat{H R}$ & فاطميند & $\widehat{\beta}$ & فاطمينان & $\hat{O R}$ & فاصله & $\hat{\beta}$ & \\
\hline- & - & - & - & - & - & - & - & $\begin{array}{l}* / P 9) \\
(* / 18\end{array}$ & . $/$ TA & $\begin{array}{l}-\cdot / M) \\
(-V / A r\end{array}$ & $-1 / \pi v$ & ثابت هدل \\
\hline $\begin{array}{l}1 / \text { (1) } \\
(\cdot / 91,\end{array}$ & I/IV & $\begin{array}{l}. / M T) \\
(-.1 .9\end{array}$ & .118 & $\begin{array}{l}1 / 99) \\
(. / 41\end{array}$ & $1 / \Delta 1$ & $\begin{array}{l}+189) \\
(-.18 V\end{array}$ &. $\mid+1$ & $\begin{array}{l}V \cdot r) \\
(\cdot / \Delta r\end{array}$ &.$/ 199$ & $\begin{array}{l}+/+Y) \\
(-. / \& T,\end{array}$ &.$- / M$ & جنس \\
\hline $\begin{array}{l}\text { l/eq } \\
(* / q \varepsilon\end{array}$ & We & $\begin{array}{l}-* / H A) \\
(-* / * t\end{array}$ & .110 & $\begin{array}{l}V / r \cdot) \\
(* / r \Delta\end{array}$ & . pes & $\begin{array}{l}\cdot / M) \\
(-1 / 4 \cdot\end{array}$ & $-\cdot / W$ & $\begin{array}{l}r / A r) \\
(r / \bullet \Delta,\end{array}$ & $r / I r$ & $(\cdot M r, 1 / \cdot P)$ & -NO & خابقه فشار \\
\hline $\begin{array}{l}(1 /)) \\
(.189\end{array}$ & $V / v$ & $\begin{array}{l}. / 19) \\
(-. / T V\end{array}$ & 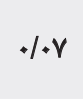 & $\begin{array}{l}1 / m c) \\
(\cdot / R E\end{array}$ & $\cdot / v$ & $\begin{array}{l}+/ T q) \\
(-* / N A\end{array}$ & - & $\begin{array}{l}\Delta / T V) \\
(1 / T \cdot,\end{array}$ & T/Tr & $(\cdot / 1 \wedge, 1 / \& A)$ & W/Y & سكته قلبيى \\
\hline $\begin{array}{l}\text { YH.) } \\
(\cdot N A\end{array}$ & $11 \cdot 9$ & $\begin{array}{l}\left.\cdot / M^{q}\right) \\
(-. / 49\end{array}$ & .1 .9 & $\begin{array}{l}V(\Delta P) \\
\left(\cdot / T^{+}\right.\end{array}$ & . / & $\begin{array}{l}. / N 4) \\
(-* / 48\end{array}$ & $-* / \cdot v$ & $\begin{array}{l}r / E V) \\
(1 / R V\end{array}$ & T/ge & $(\cdot(\Delta), 1 / r+)$ & $.14 \mathrm{~A}$ & سابقه \\
\hline $\begin{array}{l}r / A Y) \\
(1 / A,\end{array}$ & V/AF & $(\cdot 1 \cdot 1,1 / \cdot 1)$ & .181 & $\begin{array}{l}1 / 94) \\
(\cdot / \Delta \Delta,\end{array}$ & $1 / A 1$ & $\begin{array}{l}.189) \\
(-. / 109\end{array}$ & .108 & $\begin{array}{l}\text { V/TE } \\
(\cdot / T Q\end{array}$ &.$/ N$ & $\begin{array}{l}+/ \pi r) \\
(-1 / f \cdot,\end{array}$ &.$- / 48$ & كمليوى \\
\hline $\begin{array}{l}R / P T) \\
(T / T E\end{array}$ & r/W & $(\cdot / W, V / M T)$ & $1 / r$ & $\begin{array}{l}r / 8+4) \\
(1 / \cdot 9,\end{array}$ & $M / M$ & $\begin{array}{l}1 / 79) \\
(* 1+9\end{array}$ &.$N A$ & $\begin{array}{l}1 / 8 \cdot) \\
(\cdot / M\end{array}$ &.$/ 9$. & $\begin{array}{l}\cdot / m y) \\
(-\cdot / N r\end{array}$ & $-* /$ & سن \\
\hline $\begin{array}{l}\cdot(A)) \\
(\cdot / R A\end{array}$ &.$/ 94$ & $\begin{array}{l}-+/ T I) \\
(-\cdot M r\end{array}$ & $-+/ 4 \Delta$ & $\begin{array}{l}\left(1 / T^{4}\right) \\
\left(\cdot / F^{e}\right)\end{array}$ & $\cdot / N^{2}$ & $\begin{array}{l}+/ 4 q) \\
(-\bullet / 14\end{array}$ & $-+/ M T$ & $\begin{array}{l}V / M T) \\
(\cdot N 8,\end{array}$ & $1 / 19$ & $\begin{array}{l}+/ T A) \\
(-* / T V\end{array}$ &.$/ \mathrm{V}$ & تهيزان \\
\hline $\begin{array}{l}m / \circ q) \\
(1 / r q\end{array}$ & The & $(\cdot / \pi T, N / M)$ & $\cdot M$ & $\begin{array}{l}1 / 4 q) \\
(\cdot / N \Delta\end{array}$ & $1 / \cdot$ & $\begin{array}{l}\cdot / 4 \cdot) \\
(-+/ 4 q\end{array}$ & $.1 .0 \mathrm{rg}$ & $\begin{array}{l}r / N S) \\
(\cdot / T V\end{array}$ & 111 & $\begin{array}{l}1 / r \Delta) \\
(-1 / / r)\end{array}$ & .11 & 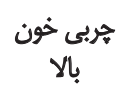 \\
\hline
\end{tabular}

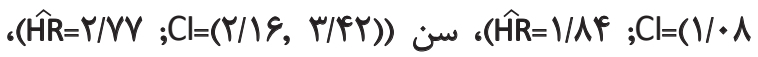

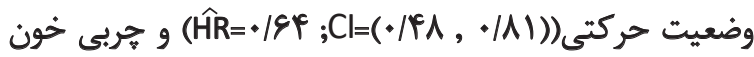

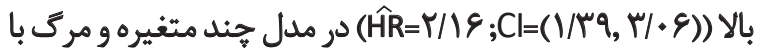

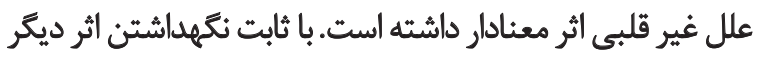

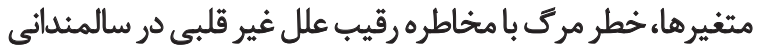

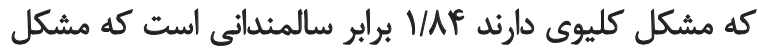

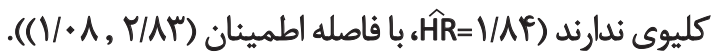

بحث

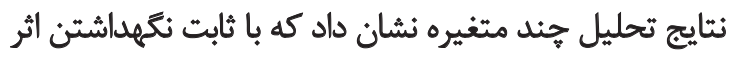

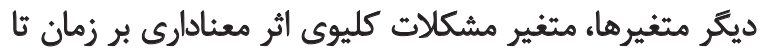

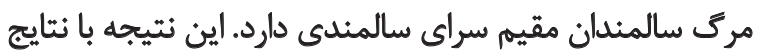

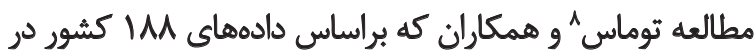

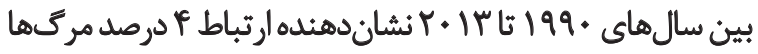

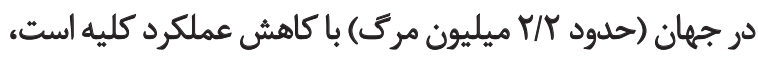

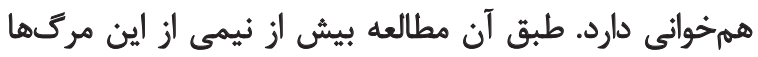

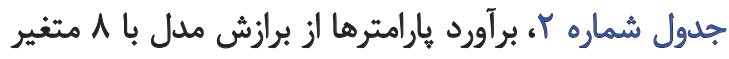

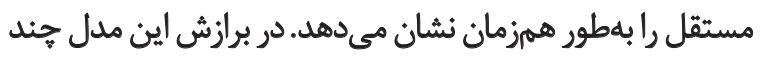

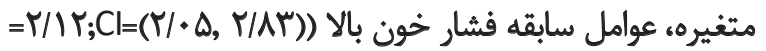

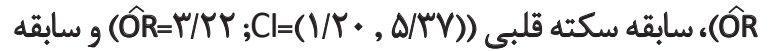

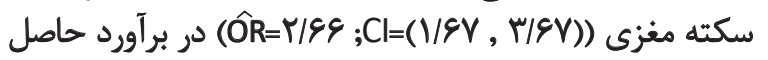

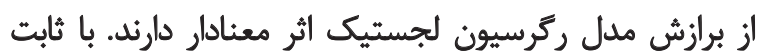

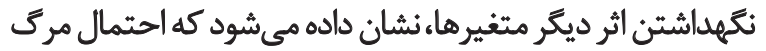

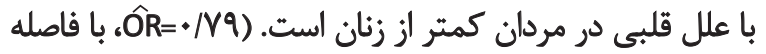

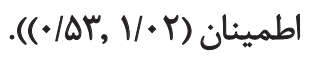

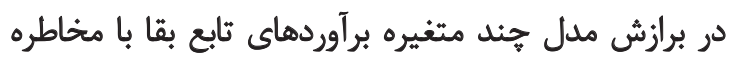

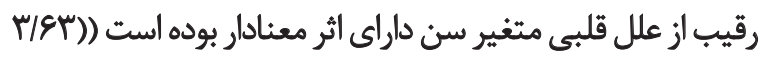
(HR= Y/MA ;Cl=(1/.9 ,

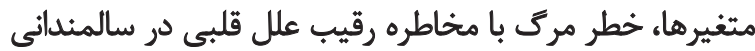

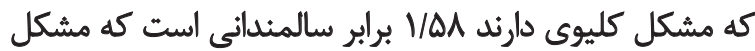

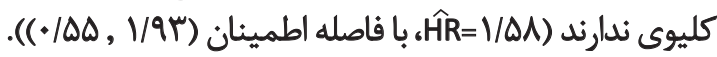

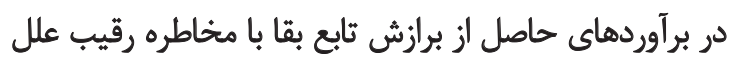

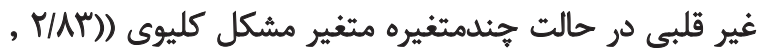


افزايش زمان بقاى سالمندان عوامل مذكور نيز مدنظر قرار گيرد.

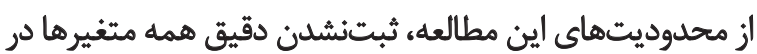

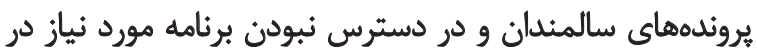

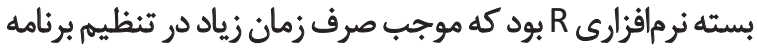

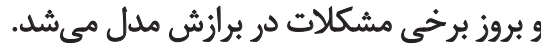

$$
\text { تشكر و قدرانى }
$$

اين مقاله بركرفته از يايان إمه مقطع كارشناسى ارشد آمار

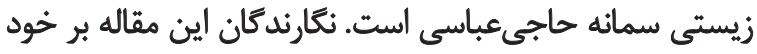

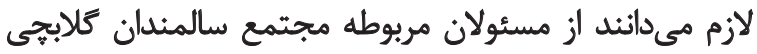

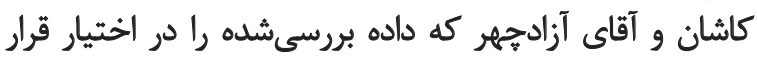

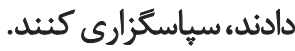

(1/T ميليون مورد) مرتبط با قلب بود، در حالى كه در حدود

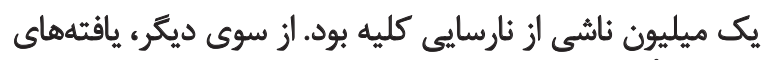

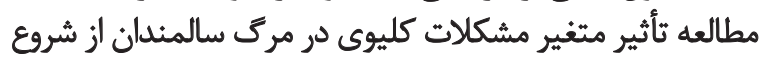

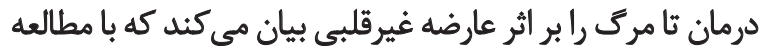

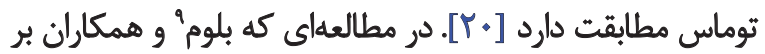

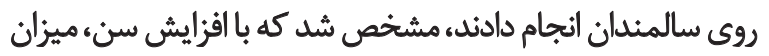

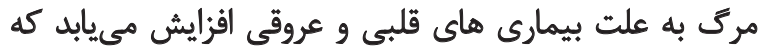

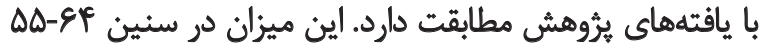
سال، \&D-VF سال و VD

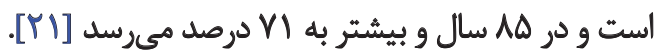
در تحليلهاى تكمتغيره، اثر معنادار سابقه سكته مغزى بر بر بران

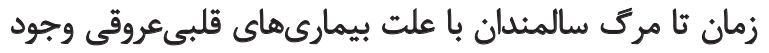

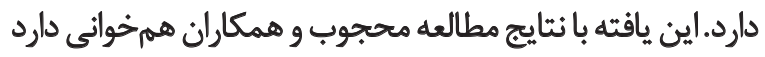

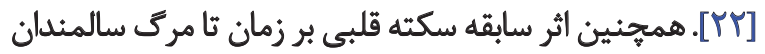

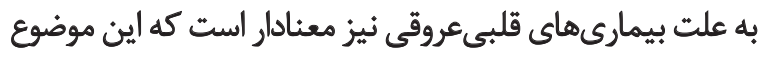

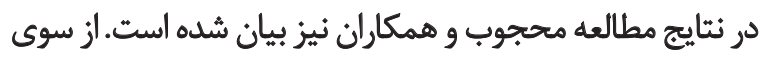

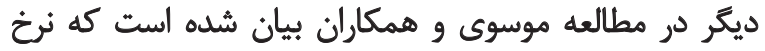

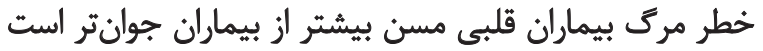

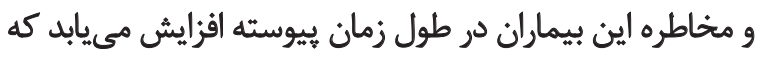

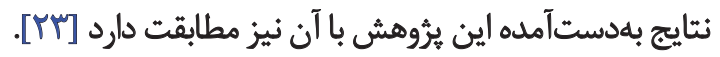
شريفنيا و همكاران در مطالعهاى كه روى 199 بيمار قلبي

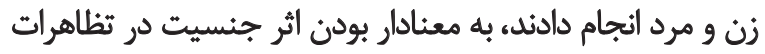

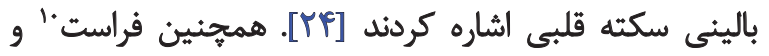

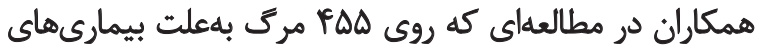

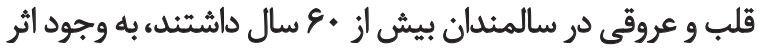

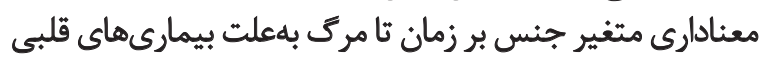

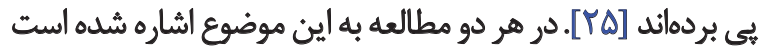

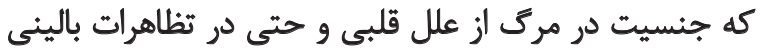

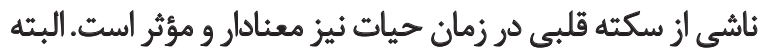

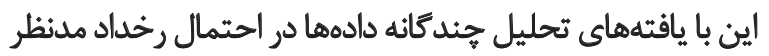

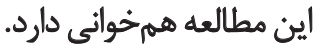

\section{نتيجهيَيرى نهايى}

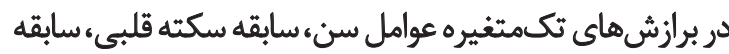

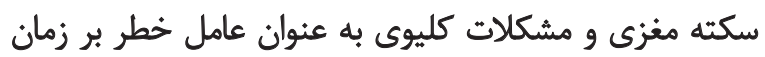

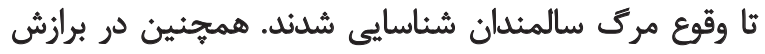

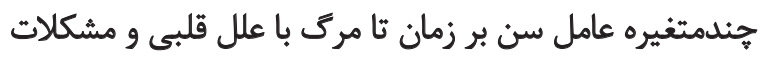

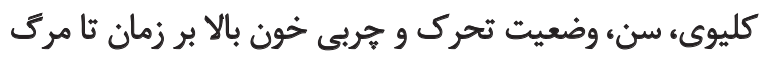

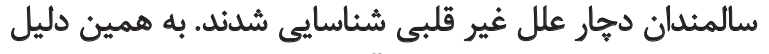

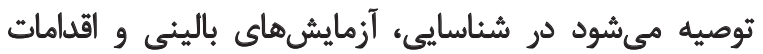

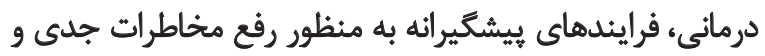

9. Blom JW

10. Frost 


\section{References}

[1] Ameri G, Govari F, Nazari T, Rashidinejad M, Afsharzadeh P. [The adult age theories and definitions (Persian)]. Hayat. 2002; 8(1):4-13.

[2] Farzianpour F. Arab M. Foroushani A. Morad Zali E. Evaluation of the criteria for quality of life of elderly health care centers in Tehran Province, Iran. Global Journal of Health Science. 8(7):6876. doi: 10.5539/gihs.v8n7p68

[3] Goharinezhad S. Maleki M. Baradaran HR, Ravaghi H. A qualitative study of the current situation of elderly care in Iran: what can we do for the future? Global Health Action. 2016; 9(1):32156. doi: $10.3402 /$ gha.v9.32156

[4] Noroozian M. The Elderly population in Iran. An ever growing concern in the health system. Iranian Journal of Psychiatry and Behavioral Sciences. 2012; 6(2):1-6.

[5] Carroll W, Miller GE. Heart disease among elderly Americans. Estimates for the U.S. Civilian Noninstitutionalized Population, Statistical Brief 409. Rockville: Agency for Healthcare Research and Quality; 2013

[6] Farkhani EM, Baneshi MR, Zolala F. [Survival rate and its related factors in patients with acute myocardial infarction (Persian)]. Medical Journal of Mashhad University of Medical Sciences. 2014; 57(4):636-46

[7] Beyranvand MR, Lorvand A, Alipour Parsa S, Motamedi MR, Kolahi AA. [The quality of life after first acute myocardial infarction (Persian)]. Pajoohande. 2011; 15(6):264-72.

[8] Andrawes WF, Bussy C, Belmin J.. Prevention of cardiovascular events in elderly people. Drugs \& Aging. 2005; 22(10):859-76. doi: 10.2165/00002512-200522100-00005

[9] Sharifirad GhR. Mohebbi S. Matlabi M. [The relationship of physical activity in middle age and cardiovascular problems in old age in retired people in Isfahan, 2006 (Persian)]. Ofogh-eDanesh. 2007; 13(2):57-63.

[10] Taghavi M. [Mortality rate in 23 province of the country in 2003 (Persian)]. Tehran: Ministry of Health; 2005.

[11] Parkash R, Choudhary SK, Singh US. A study of morbidity pattern among geriatric population in an urban Area of Udaipur Rajasthan. 2004; 29(1):35-40.

[12] Haller B. The analysis of competing risks data with a focus on estimation of cause-specific and sub-distribution hazard ratios from a mixture model [PhD thesis]. München: University of München; 2014.

[13] Choi S, Huang X. Maximum likelihood estimation of semiparametric mixture component models for competing risks data. Biometric Methodology. 2014; 70(3):588-98. doi: 10.1111/biom.12167

[14] McLachlan G, Peel D. Finite mixture model. Hoboken, New Jersey: John Wiley \& Sons; 2000.

[15] Abu Bakar MR, Daud I, Ibrahim NA, Rahmatina D. Estimating a Logistic Weibull Mixture Models with Long-Term Survivors. Jurnal Teknologi. 2006;45(1):57-66. doi: 10.11113/jt.v45.323

[16] Azadchehr M, Rahgozar M, Karimloo M, Adib Haj Bageri M. [To identify some factors effective on survival of the elderly living in nursing home using Copula Competing Risk Model: Bayesian approach (Persian)]. Journal of Health Promotion Management. 2014; 3(4):46-55

[17] Schlattmann P. Medical application of finite mixture model. Berlin: Springer; 2009.

[18] Kuk AYC. A semi-parametric mixture model for the analysis of competing risks data. Australian Journal of Statistics. 1992; 34(2):169-80. doi: 10.1111/j.1467-842x.1992.tb01351.x

[19] Ng SK, McLachlan GJ. An EM-based semi-parametric mixture model approach to the regression analysis of competing-risks data. Statistics in Medicine. 2003; 22(7):1097-111. doi: 10.1002/ sim.1371

[20] Thomas B, Matsushita K, Abate KH, Al-Aly Z, Ärnlöv J, Asayama K et al. Global cardiovascular and renal outcomes of reduced GFR. Journal of the American Society of Nephrology. 2017; 28(7):2167-79. doi: 10.1681/ASN.2016050562.

[21] Mahjoub H, Rusinaru D, Soulière V, Durier C, Peltier M, Tribouilloy $\mathrm{C}$. Long-term survival in patients older than 80 years hospitalised for heart failure. A 5-year prospective study. European Journal of Heart Failure. 2008; 10(1):78-84. doi: 10.1016/j. ejheart.2007.11.004

[22] Mosavy S, Soltanian AR, Roshanaei GH, Fardmal J. [Applying Aalen's additive hazard model for analyzing 5-year survival of acute myocardial infarction patients in Bushehr (Persian)]. Journal of North Khorasan University of Medical Science. 2011; 3:16169.

[23] Nazari R, Haghdost AA, Rezaie R, Sa'atsaz S, Chan YH et al, Difference in clinical symptoms of myocardial infarction between men and women. Iranian Journal of Critical Care Nursing. 2011; 2011; 4(1):e7081

[24] Frost PH, Davis BR, Burlando AJ, David Curb J, Guthrie GP, Isaacsohn JL, et al. Coronary heart disease risk factors in men and women aged 60 years and older findings from the systolic hypertension in the elderly program. Circulation. 1996; 94(1):26-34. doi 10.1161/01.cir.94.1.26

[25] Frost PH, Davis BR, Burlando AJ, Curb JD, Guthrie GP, Isaacsohn JL, et al. Coronary heart disease risk factors in men and women aged 60 years and older: indings from the systolic hypertension in the elderly program. Circulation. 1996; 94(1):26-34. doi: 10.1161/01.cir.94.1.26 


\section{SALMAND: \\ IRANIAN JOURNAL of AGEING \\ volume 12, Number 4, Winter 2017}

Table of Contents

402 Effect of Physical Activity on Cognitive Flexibility and Perfectionism in the Elderly

414 Determining Psychometric Properties of Iranian Active Aging Measurement Instrument

430 Identification of Facilitators and Deterrents of the Quality of Life in Elderly Women and Men: A Phenomenological Research

446 Relationship Between Styles of Attachment to God and Death Anxiety Resilience in the Elderly

458 The Relationship Between Personality Traits (Neuroticism and Extraversion) and Self-Efficacy With Aging Depression

470 Relationship Between Self-Regulation and Balance-Confidence in Active and Inactive Elderly Men

482 Effect of Group Logotherapy on Life Expectancy and Mental and Social Wellbeing of The Female Elderly Residents of Nursing Homes in Dubai

494 Relationship Between General Health and Demographic Characteristics of Family Caregivers of Stroke Survivors

506 Adjustment of Optimal Sports Site Selection Criteria for Elderly Using Analytical Hierarchy Process and Geographic Information System

518 Identifying Some Risk Factors for the Time to Death of the Elderly Using the Semi-Parametric Blended Model of Survival Analysis With Competing Risks
Entezari M, Shamsipour Dehkordi P, Sahaf R.

Mohammadi E, Allahyari T, Darvishpoor Kakhaki A, Saraei H.

Imanzadeh A, Hamrahzdeh M.

Bitarafan L, Kazemi M, Yousefi Afrashte M.

Abbasi M, Mirderikvand F, Adavi H, Hojati M.

Sadat Hosseini F, Hatamnezhad O.

Saffarinia Afsaneh Dortaj M.

Tajvidi M, Dalvandi A, Sahaf R, Rahgozar M.

Jamshidi O, Doostipasha M, Razavi S M H, Gudarzi M.

Hajiabbasi S, Rahgozar M, Biglarian A, Jalali A, Azadchehr MJ. 


\section{Hamid Reza Khankeh, PhD}

PhD in Nursing, Department of Nursing, University of Social Welfare and Rehabilitation Sciences, Tehran, Iran.

Post-Doctoral Research Fellowship in Health in Emergency and Disaster, Department of Health in Disasters and Emergencies, University of Social Welfare and Rehabilitation Sciences, Tehran, Iran.

Department of Clinical Science and Education, Karolinska Institutet, Sweden.

Department of Educational and Rehabilitation Psychology, Leipzig

University, Leipzig, Germany.

Hamid Shokoohi, MD, MPH

Assistant Professor, Emergency Medicine, Emergency Ultrasound Fellowship, Department of Emergency Medicine, School of Medicine and Health Sciences, George Washington University, Washington, US.

\section{Mohammad Mohammad-Zadeh, PhD}

Assistant Professor, PhD in Physiology, Cellular and Molecular Research Center, Sabzevar University of Medical Sciences, Sabzevar, Iran.

\section{Abolghasem Pourreza, PhD}

PhD in Health Economics, Department of Health Management and Economics, School of Public Health and Health Services, Tehran University of Medical Sciences, Tehran, Iran.

\section{Mehdi Rassafiani, PhD}

Assistant Professor, PhD in Occupational Therapy, Department of Occupational Therapy, University of Social Welfare and Rehabilitation

$$
\text { Sciences, Tehran, Iran. }
$$

Iranian Research Center on Aging, University of Social Welfare and

$$
\text { Rehabilitation Sciences, Tehran, Iran. }
$$

Health Sciences Research Center, Kuwait University, Kuwait, Kuwait.

\section{Manouchehr Ardjomand Hessabi, MD, MPH}

Center for Clinical and Translational Sciences, University of Texas,

$$
\text { Austin, Texas. }
$$

Health Science Center at Houston, Houston, Texas.

\section{Ahmad Delbari, MD, PhD}

Assistant Professor, PhD in Clinical Geriatric and Neuroscience,

Iranian Research Center on Aging, University of Social Welfare and Rehabilitation Sciences, Tehran, Iran.

Division of Clinical Geriatrics, Department of Neurobiology, Care

Sciences and Society, Karolinska Institute, Huddinge Hospital, Stockholm, Sweden.

\section{Reza Salman Roghani, MD}

Assistant Professor, Physical Medicine and Rehabilitation, Department of Clinical Sciences, University of Social Welfare and Rehabilitation Sciences, Tehran, Iran.

\section{Shima Sum, PhD}

Assistant Professor, PhD in Gerontology, Department of Social Medicine, Faculty of Medicine, Babol University of Medical Sciences, Babol, Iran.

\section{Masoud Fallahi Khoshknab, PhD}

Assistant Professor, PhD in Nursing, Department of Nursing, University of Social Welfare and Rehabilitation Sciences, Tehran, Iran.

\section{Neda Mehrdad, PhD}

PhD in Nursing, Elderly Health Research Center, Endocrinology and Metabolism Population Sciences Institute, Tehran University of Medical Sciences, Tehran, Iran.

Endocrinology and Metabolism Research Center, Endocrinology and Metabolism Clinical Sciences Institute, Tehran University of Medical Sciences, Tehran, Iran.

\section{Asghar dadkhah, PhD}

Assistant Professor, PhD in Psychology, Department of Psychology, University of Social Welfare and Rehabilitation Sciences, Tehran, Iran.

\section{Hashem Shemshadi, MD}

Assistant Professor, Plastic and Reconstructive Surgery, Department of Clinical Sciences, University of Social Welfare and Rehabilitation Sciences, Tehran, Iran.

\section{Ahmad-Ali Akbari Kamrani, MD}

Internal Medicine, Fellowship in Geriatrics, Iranian Research Center on Aging, University of Social Welfare and Rehabilitation Sciences, Tehran, Iran.

\section{Hafiz TA. Khan, PhD}

School of Health Sciences, Faculty of Health, Education and Life Sciences, Birmingham City University, Westbourne Road, Edgbaston, Birmingham, UK.

\section{Robab Sahaf, MD, PhD}

Assistant Professor, PhD in Gerontology, Iranian Research Center on Aging, University of Social Welfare and Rehabilitation Sciences, Tehran, Iran

\section{Reza Fadayevatan, PhD}

PhD in Gerontology, Iranian Research Center on Aging, University of Social Welfare and Rehabilitation Sciences, Tehran, Iran.

\section{Mahshid Foroghan, MD}

Assistant Professor, Psychiatry Specialist, Geriatric Psychiatry Fellowship, Iranian Research Center on Aging, University of Social Welfare and Rehabilitation Sciences, Tehran, Iran.

\section{Farahnaz Mohammadi, PhD}

Assistant Professor, PhD in Nursing, Department of Nursing, University of Social Welfare and Rehabilitation Sciences, Tehran, Iran.

\section{Parisa Taheri Tanjani, MD}

Geriatric Medicine, Department of Internal Medicine, Ayatollah Taleghani Hospital Research Development Unit, Shahid Beheshti University of Medical Sciences, Tehran, Iran.

Charbanou Jochum-Maghsoudnia, PhD Professor in Geriatrics, University of Rennes, Rennes, France. 


\section{Members of Editorial Board (Alphabetic Order)}

\section{Fati Nourhashémi, MD}

Professor in Geriatrics, Universite Paul Sabatier Toulouse III, Institute of Aging, Toulouse, France

Hossein Najmabadi, PhD

PhD in Molecular Biology, Genetics Research Center, University of Social Welfare and Rehabilitation Sciences, Tehran, Iran.

\section{Muhammad Kamalinejad, PhD}

School of Pharmacy martyr Beheshti University of Medical Sciences, Tehran, Iran.

Pedram Ghafourifar, PhD

Assistant Professor, Pharmacologist. Tri-State Institute of Pharmaceutical Sciences, Huntington, US.

\section{Kimia Kahrizi, MD}

Professor of Pediatrics, Clinical Genetics. Genetics Research Center, University of Social Welfare and Rehabilitation Sciences, Tehran, Iran.

\section{Taher Darreh-Shori, PhD}

Assistant Professor, Pharmacologist, Alzheimer Neurobiology Center, Department of Neurobiology, Care Sciences and Society, Karolinska Institute, Stockholm, Sweden.

\section{Mahyar Salavati, PhD}

Assistant Professor, PhD in Physiotherapy, Professor of Physiother apy Department, University of Social Welfare and Rehabilitation Sciences, Tehran, Iran.

Mohammadreza Soroush, MD, MPH

Assistant Professor, Janbazan Medical and Engineering Research Center (JMERC), Tehran, Iran.

\section{Azita Emami, PhD}

PhD in Nursing. Seattle University, Seattle, Washington, US.

\section{Hamid Reza Khorram Khorshid, MD, PhD}

PhD in Medical Genetics, Genetics Research Center, University of Social Welfare and Rehabilitation Sciences, Tehran, Iran.

\section{Seyed-Mohammad Fereshtehnejad, MD, PhD}

Assistant Professor, Postdoctoral Research Fellow, Department of Neurology and Neurosurgery, McGill University, Montreal, Canada. Division of Clinical Geriatric, Department of Neurobiology, Care Sciences and Society (NVS), Karolinska Institute, Stockholm, Sweden.

\section{Mina Ohadi, MD, PhD}

PhD in Medical Genetics, Iranian Research Center on Aging, University of Social Welfare and Rehabilitation Sciences, Tehran, Iran.

\section{Alireza Mosavi Jarrahi, PhD}

Assistant Professor, PhD in Epidemiology. Medical School, Shahid Beheshti University of Medical Sciences, Tehran, Iran.

\section{Mohammad Ali Mohseni-Bandpei, PhD}

Phd in Physiotherapy, Department of Physiotherapy, University of Social Welfare and Rehabilitation Sciences, Tehran, Iran. Genetics Research Center, Tehran, Iran.

Visiting Professor, University Institute of Physical Therapy, Faculty of Allied Health Sciences, University of Lahore, Lahore, Pakistan.

\section{Farid Najafi, MD, PhD}

PhD in Epidemiology, Kermanshah Epidemiology Research Center (KEERC), Kermanshah University of Medical Sciences, Kermanshah, Iran.

\section{Maryam Noroozian, MD}

Department of Memory and Behavioral Neurology, Tehran University of Medical Sciences, Tehran, Iran.

Department of Psychiatry, Tehran University of Medical Sciences, Tehran, Iran.

Tengku Aizan Hamid, PhD

Malaysian Research Institute on Ageing (MyAgeing), Universiti Putra Malaysia, Seri Kembangan, Malaysia.

\section{Martin Mortazavi, MD}

Neurological Surgeon, fellowship Trained in Cerebrovascular and Skull Base Surgery, Division of Neurological Surgery, The University of Alabama at Birmingham (UAB), US.

Yadollah Abolfathi Momtaz, PhD

PhD in Gerontology, Iranian Research Center on Aging, University of Social Welfare and Rehabilitation Sciences, Tehran, Iran. Malaysian Research Institute on Ageing (MyAgeing), Universiti Putra Malaysia, Seri Kembangan, Malaysia.

\section{Mehdi Rahgozar, PhD}

Assistant Professor, PhD in Biostatistics. Department of Biostatistics and Computer, University of Social Welfare and Rehabilitation Sciences, Tehran, Iran.

\section{Fariba Kolahdooz, PhD}

Indigenous and Global Health Research Group, Faculty of Medicine and Dentistry, Department of Medicine, University of Alberta, Canada.

\section{Seyed Kazem Malakouti, MD}

Assistant Professor, Specialist Psychiatry, Geriatric Psychiatry Fellowship, Division of Suicide Behaviors Study and Prevention WHO Collaborating Center in Mental Health, Geneva, Switzerland. School of Behavioral Sciences and Mental Health, Iran University of Medical Sciences, Tehran, Iran.

Hamid Allahverdipour, PhD

PhD in Health Education and Promotion, Department of Health Education \& Promotion, Faculty of Health, Tabriz University of Medical Sciences, Tabriz, Iran. 


\section{SALMAND: \\ IRANIAN JOURNAL of AgEING}

Iranian Journal of Ageing

Quarterly Journal of Iranian Research Center on Ageing

Volume 12, Number 4, Winter 2018

ISSN 1735-806X

Owner: University of Social Welfare \& Rehabilitation Sciences

Chair Manager: Dr. Ahmad Ali Akbari Kamrani

Chief Editor: Dr. Robab Sahaf

Executive Director: Dr. Yadollah Abolfathi Momtaz

Scientific Directors: Vida Alizad, Parisa Ali Hasanzadeh

HEAD of Editorial Board: Dr. Hamid Reza Khorram Khorshid

Publisher: Negah Institute for Scientific Communication

www.nirp.ir

Tel \& Fax:

+98 (21)86036497

Proof Reading Editors:

Vahideh Towhidlou, Mina Amani, Somayeh Salimian

Graphics:

Fatemeh Rezaee

Web Master:

Atabak Sarbakhsh

Subscription

Iranian Ageing Magazine will be freely sent to the libraries, research and scientific institutes related to the universities though out the counter. The real persons applying for the magazine can send their request to the office of magazine to receive it.

Address: Salmand Journal Office, University of Social Welfare \& Rehabilitation Sciences, Kudakyar St. Daneshjoo Blvd. Evin, Tehran, Iran.

Tel: +98 (21)22180083, 92 (Ext:2816)

Email: salmand2010@gmail.com

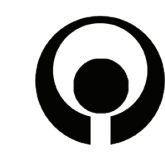

University of Social Welfare \& Rehabilitation

\section{A GEING}

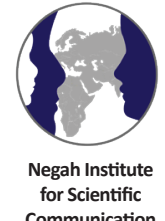

